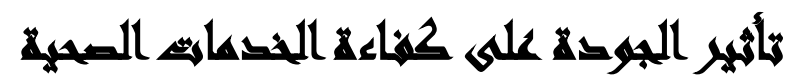

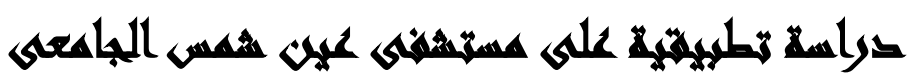

\section{[r)]}

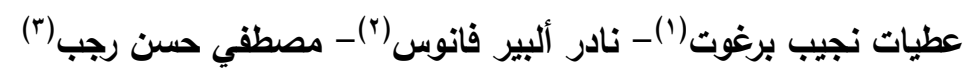

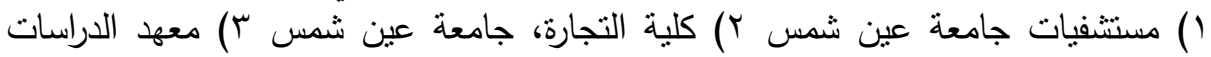
والبحوث البيئية، جامعة عين شمس عامس تُعس

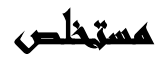

تهدف هذه الاراسة الى معرفة مدي تطبيق منهج إدارة الجودة الثاملة في نوفير وترقية

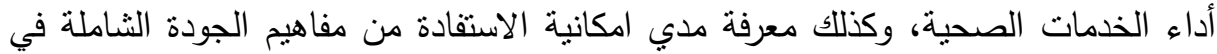

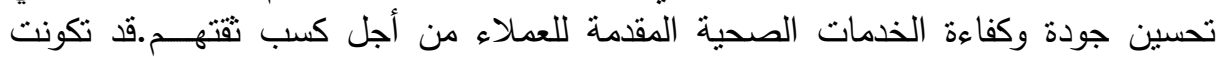

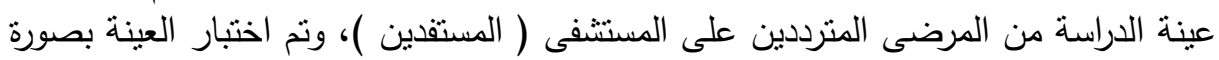

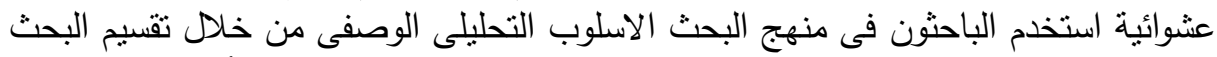

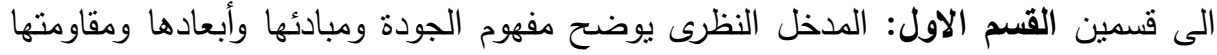
من خلال الكتب والدوريات العربية والاجنبية.

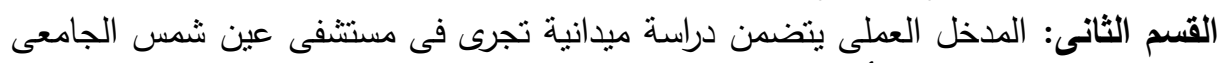

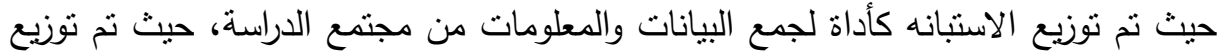

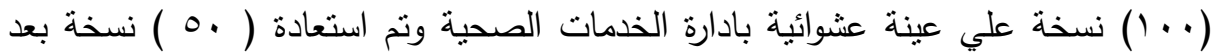

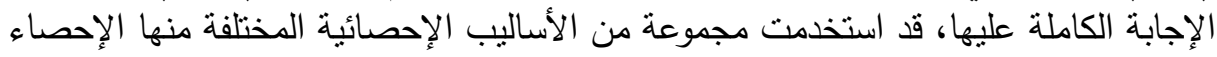

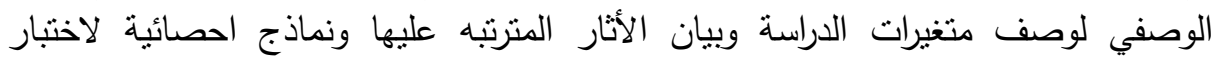

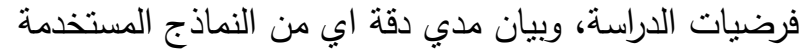

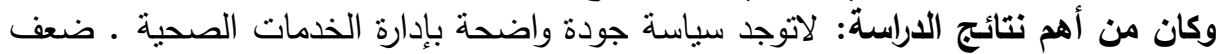

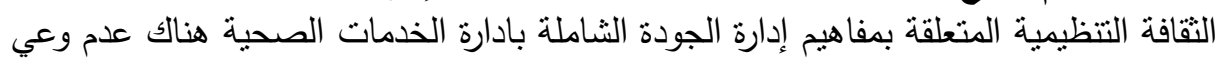

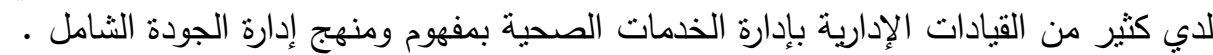

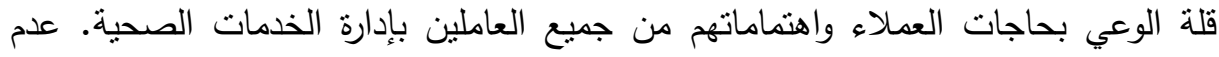

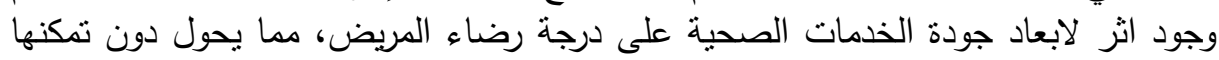
من السيطرة علي برنامج الجودة الثاملة بإدارة الخدمات الصحية. 


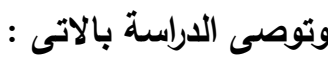

العمل بمعايير الجودة الثاملة وتطبيقاتها بتقديم خدمات عالية الجودة لمواجهة التحديات والوصول لتحقيق أهداف المستشفى ورضا المستقيدين بتقديم ما يفوق نوقعاتهم وطموحاتهم، وتحقيق التنمية المستدامة.

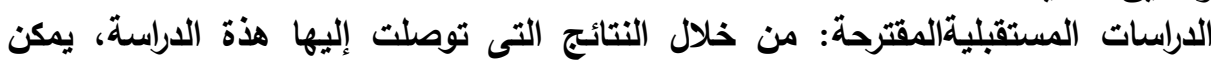
الحديث عن بعض الاتجاهات فى الاراسات التى يمكن اقتراحها مستقبلا كما يلى الئى

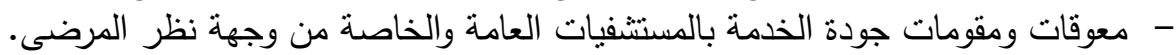

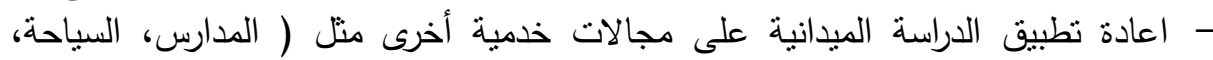

الجامعات، البنوك، الفنادق ).

\section{and}

والجدول الاتى يشمل يشهد عصرنا الحاضر الكثير من التحولات الجذرية والسريعة والتتافسية التي دفعت ولا زالت تدفع العديد من المؤسسات والمنظمات لتقديم خدمات عالية

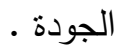

والمنظمات الصحية نواجة عدداً كبيراً من التحديات والمتغيرات البيئية وأصبحت بحاجة

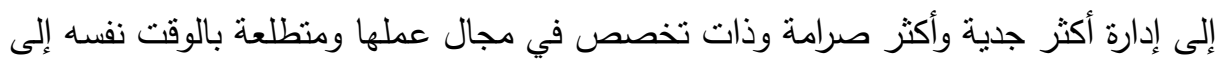

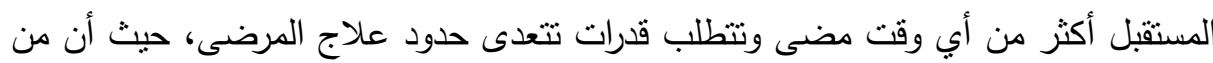

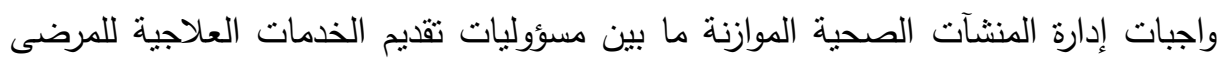

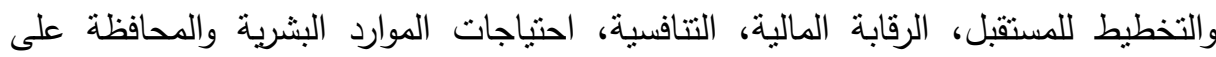
الجودة. وأن ذلك يعني إعادة النظر والتفكير بصورة جوهرية في كيفية إدارة تلك المنظمات الصحية وحول ماهية الأدوات والتقنيات التي ستنتخدها لمواجهة كل تلثك التحديات والمتغيرات، ومن بين تلك الأدوات وأكثرها أهمية هي إدارة الجودة الثاملة (TQM).

فقد أصبح التركيز على الجودة مطلبا أساسيا في مواجهة التحديات والمتغيرات المستقلية

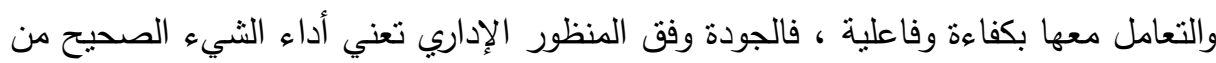

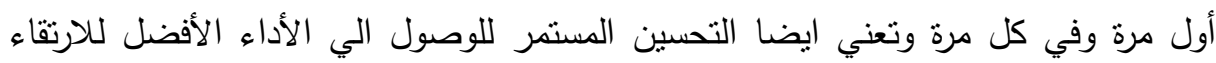

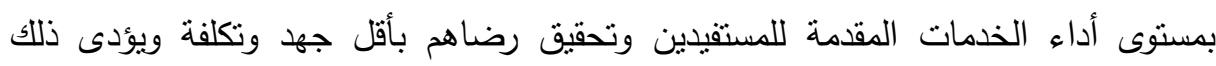

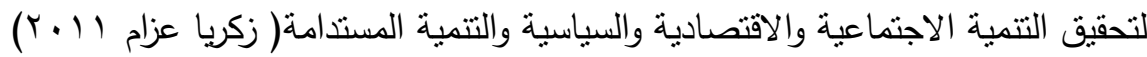
542

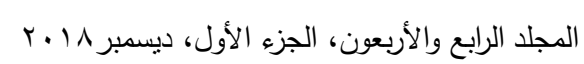




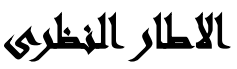

\section{Conce General مفاهيم عامة}

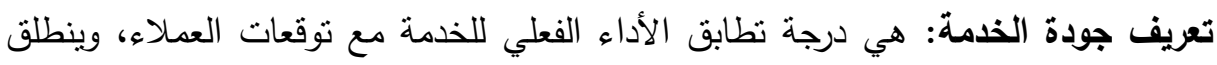

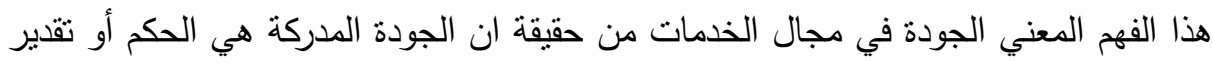

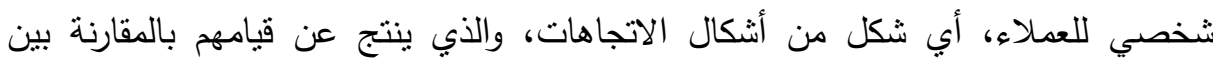
توقعاتهم للخدمة وإدراكهم للأداء الفعلي لها (المرسي والخياط ... بَ).

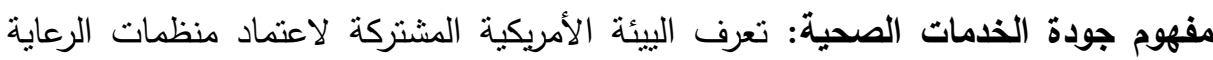

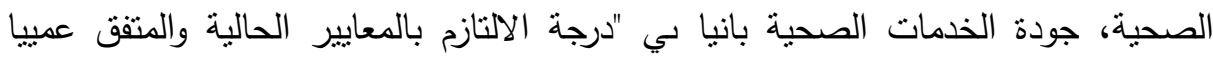

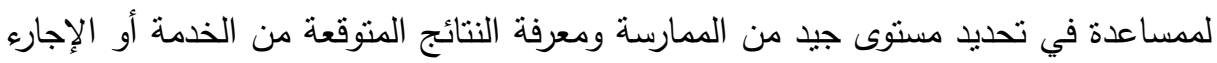

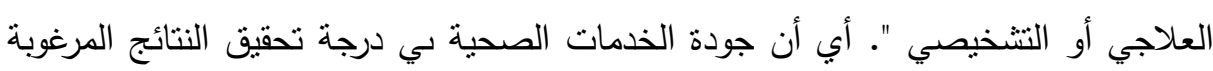
وتقميل الننائج غير المرغوبة في ظل الحالة المعرفية في فترة زمنية معينة .

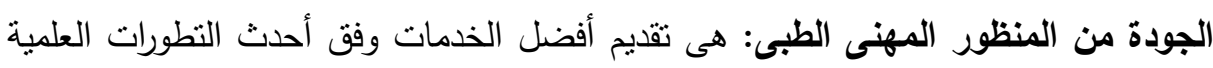

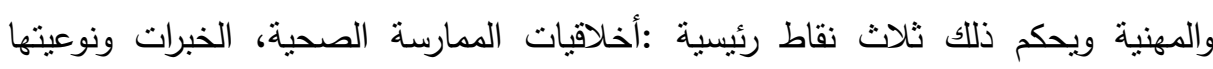

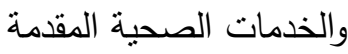

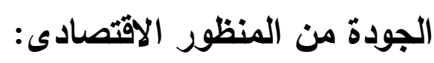
- تعنى بالدرجة الاساسية بكيفية استخدام الموارد المتوفرة والقدرة على جذب مزيد من الموارد

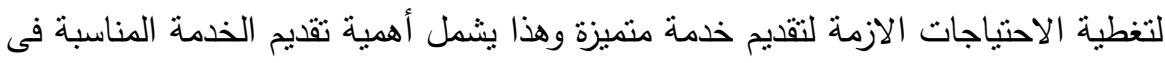

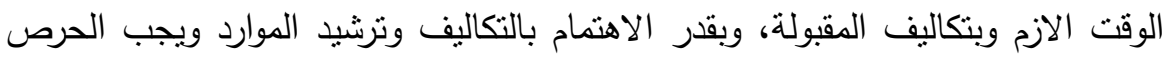
على ان لايكون ذلك على حساب الجودة فى الاداء: - ويتطلب ذلك كفاءة ادارية على المستوى التخطيطى وعلى المستوى التتفيذى ويتطلب كفاءة على المستوى الفنى وكفاءة على المستوى الثخصى وينطلب تنظيما اداريا داخليا جيد الفيد

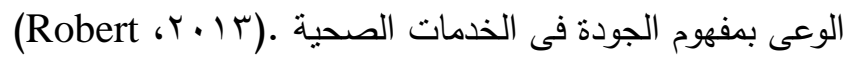




$$
\text { وتثبر الاراسات السابقة إلى: }
$$

H - ن ناك أسلوبين لقياس جودة الخدمة ينسب أولهما إلى (Berry,et.-al.,1985) وهو الذي يستتد على نوقعات العملاء لمستوى الخدمة وإدراكهم لمستوى أداء الخدمة المقدمة بالفعل، ومن ثم تحديد الفجوة ( أو التطابق ) بين هذه التوقعات والادراكات وذلك باستخدام الأبعاد الممنلة لمظاهر جودة الخدمة:

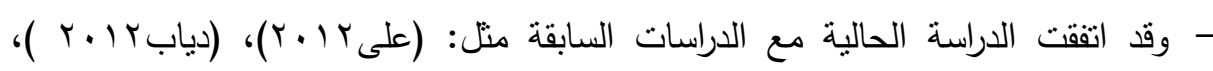

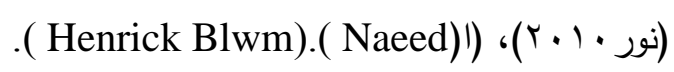

\section{مهيحلة القراسة}

تدنى مفهوم الجودة فى الخدمات الصحية ووجود ضعف وقصور فى مستوى الرعاية

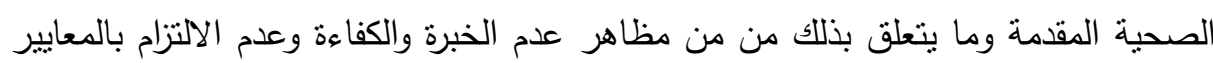

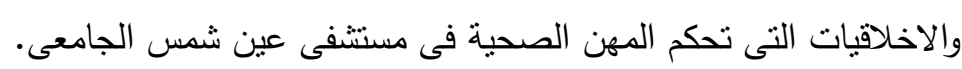

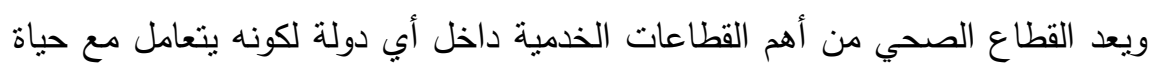

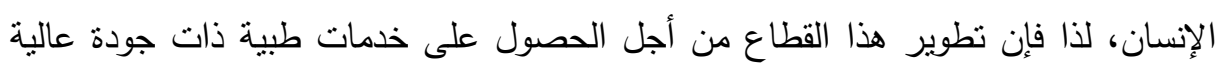
يعد هنا أستراتيجيا بالنسبة لأي دولة. وحيث أن المنظمات الصحية من مستشفيات ومراكز

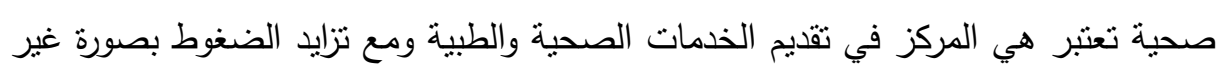

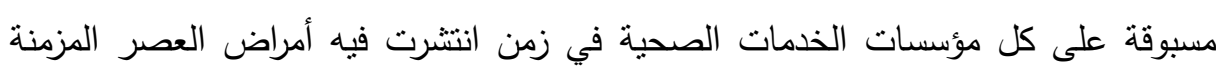
والأمراض الوبائية، فتضاعفت الضغوط المتزايدة على هذه المؤسسات الصحية والطبية، هذا بالإضافة إلى ما شهدته السنوات الأخيرة من زيادة مطردة وإقبالا متتاميا في أعداد المترددين

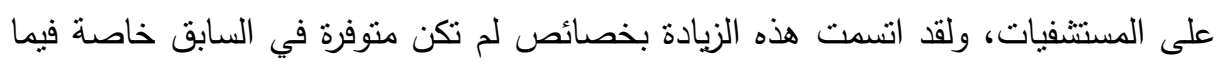

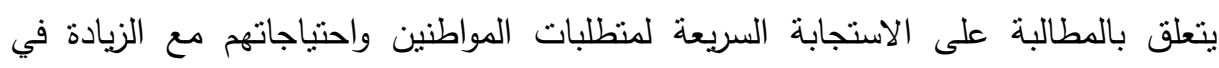
تحسين جودة الخدمات الصحية والطبية المقدمة لهم، كل ذلك حتم على القائمين على إدارة الخدمات الصحية والطبية إيجاد الوسائل والسبل لمقابلة هذه الطلبات والاستجابة لها، وأن يكون هناك تطلع دائم نحو استخدام التكنولوجيا واستحواذ كل ما هو مستحدث لتقديم الخدمات الطبية للوصول الى المستوى المطلوب لإثباع رغبات المرضى واحتباجاتهم، لذا فقد أصبح 
من الضروري تطبيق المنهج العلمي وتطبيق التطورات المذهلة في العلوم والتكنولوجيا لتحسين

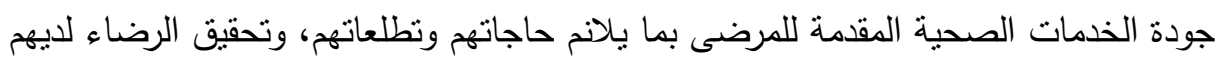
بما يحقق ميزة تتافسية في مجال صناعة الخدمات الصحية ويضمن البقاء والاستمرار داخل بلاهل السوق الصحي ( البكرى، و . . ب).

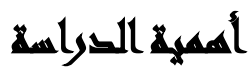

تنبع أهمية الدراسة من أهمية الجودة في الخدمة الصحية من خلال الآتي: - التوصل إلى فهم أعمق لجودة الخدمات الصحية وابعادها في المستشفى ومحاولة إبراز مساهنها فى تحقيق رضا المسفيدين (المرضى)

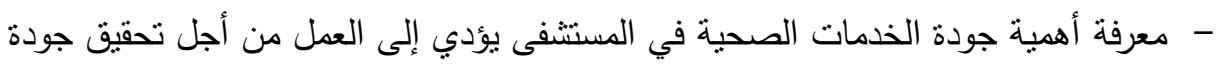
عالية في الخدمات المقدمة وتحقيق ميزة تتافسية وسمعة جيدة للمستشفى. - رجوع الافراد الاصحاء بعد شفائهم لمباشرة أعمالهم وانجازها بكفاءة يساهم فئه فئه التتمية

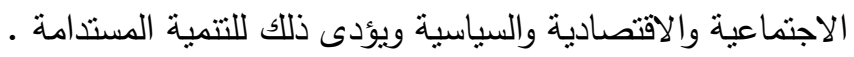

أهمبة علمية: - الرغبة فى إثراء المعرفة العلمية والاسهام فى الجهود العلمية من جانب بعض الباحثين للتوصل الى مجموعة من الحقائق التى تساعد على التعامل مع مثل هذة المشكلاء والتوصل الى نتائج ومقترحات تساهم فى دراسة وتفسير ظاهرة تدنى جودة الخدمات الصحية بالمستشفى الجامعى - محاولة التوصل الى الحلول والنتائج والمقترحات التى تساهم فى جودة وكفاءة الخدمات الصحية

\section{أهساهيم الصواسما}

تهدف هذه الدراسة إلى تقييم مستوى جودة الخدمات الصحية بالمستشفى محل الدراسة للتعرف على محددات رضاء المرضى عنها وذلك باستخدام مقياس ( SERVPERF ) 


\section{هزوض الصواسما}

الفرض الأول: لا توجد علاقة لمستوى ادراك الافراد العاملين لمفهوم واهمية الجودة للخدمات الصحية.

الفرض الثاني: لا توجد علاقة لمستوى اداء الخدمات المقدمة حالياً ومستوى الخدمات الصحية

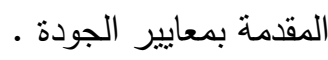

الفرض الثالث: لا نوجد علاقة لمستوى جودة وكفاءة الخدمات الصحية المقدمة ومستوى ثقة وطموحات رضا المستفدين. الفرض الرابع: لا نوجد علاقة لمستوى استخدام الموارد المادية والاجهزة والمعدات ومستوى استخدام وتطبيق طرق وادوات الجودة. الفرض الخامس: لا توجد علاقة لمستوى الحفاظ على سلامة العاملين بالمستشفى وسلامة

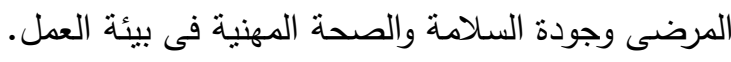

\section{الفراسايت الساريخة}

دراسة (نور، 1 م ب r) بعنوان" قياس جودة الخدمات الصحية في المستثفيات الحكومية في السودان من وجهة نظر المرضى والمراجعين. دراسة ميدانية على المستثفيات التعليمية الكبرى بولاية الخرطوم"، وهدفت إلى قياس مستوى جودة الخدمات الصحية في المستشفيات الحكومية في السودان من وجهة نظر المرضى والمراجعين. وتوصلت إلى أن هناك إدراكا ناما

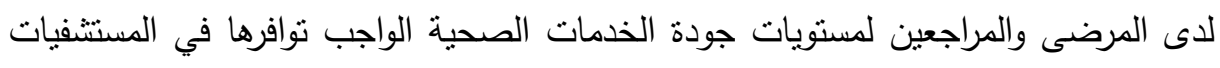
الحكومية الحى الحرفية

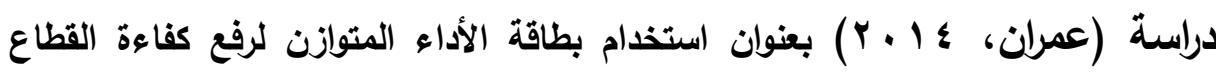

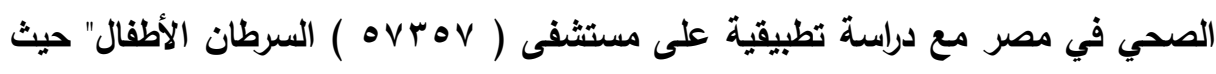

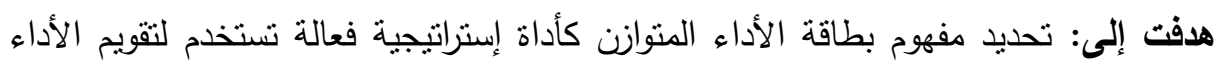




\section{وتوصلت إلى النتائج التالية}

- تعتبر بطاقة الأداء المتوازن أداة استراتيجية فعالة لقياس وتقويم أداء المنظمات المختلفة

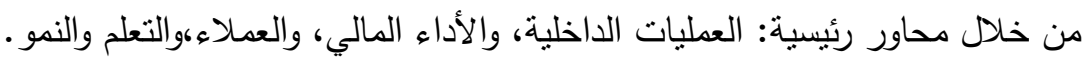

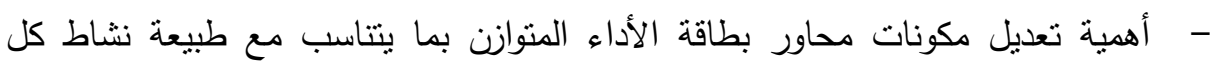

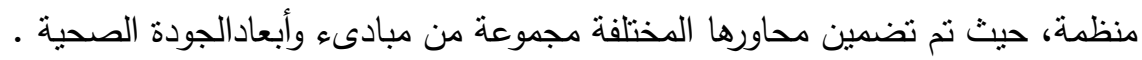

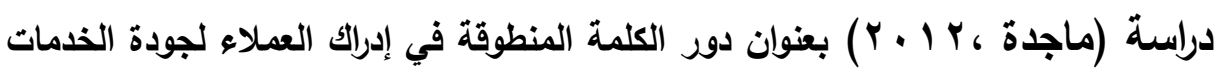
الصحية حيث هدفت إلى التعرف على دور الكلمة المنطوقة في إدراك العملاء لجودة الخدمات

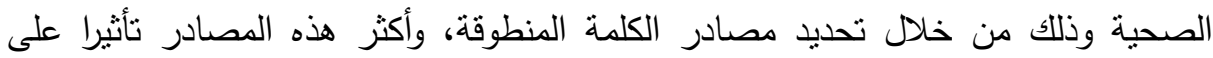
العملاء ودراسة مستوى جودة الخدمة الصحية ودرجة تاثير مصادر الكلمة المنطوقة على ودى ولى إدراك العملاء لجودة الخدمات الصحية. وتوصلت إلى وجود تأثنير معنوى لمصادر الكلمة المنطوقة على الأسرة، وفى العمل والصحف والمجلات والتليفزيون والانترنت على إدراك العملاء لجودة الخدمة الصحية . مجتمع الدراسة: مستشفيات جامعة عين شمس منهج الدراسة: لتحقيق أهداف البحث واختبار الفرضيات يتم الاعتماد على المنهج التحليلى تشعى الوصفى من خلال تقسيم البحث الى قسمين:

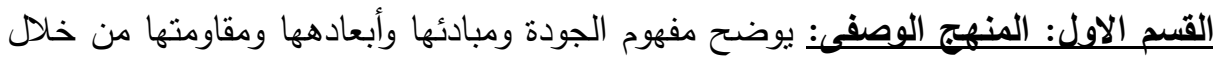
البيانات المرجعية باستخدام المنهج الوصفى كما يلى: بعض المراجع العلمية والكتب والدوريات العربية والاجنبية . القسم التانى: المدخل التطبقى: يتضمن دراسة ميدانية تجرى على مستشفى عين شمس

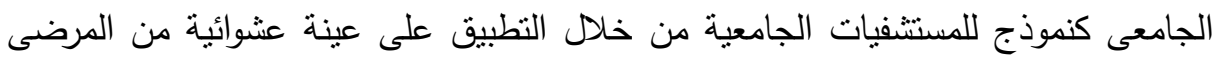

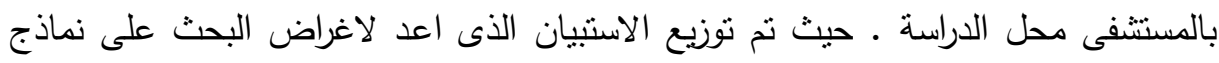

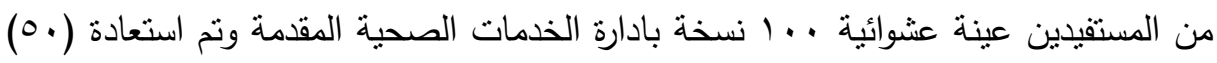
نسخة بعد الاجابة الكاملة عليها. بهدف معرفة تقييم المرضى للجودة الكلية للخدمة الصحية

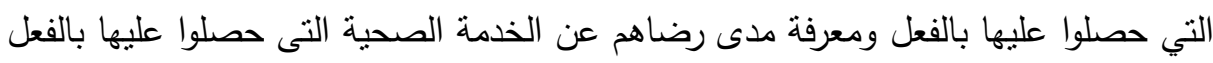

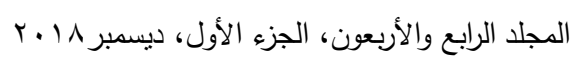


من المستشفى عين شمس الجامعى محل الدراسة وللتحقق من وجود العلاقة بين المحددات

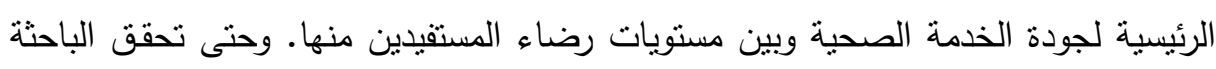
هذه الأهداف، قامت بتجميع وتصنيف بيانات الدراسة سواء النظرية أو الميدانية الأولية. بهدف التحقق من مستوى جودة الخدمات الصحية بالمستشفى باستخدام مقياس والتعرف على محددات رضاء المرضى. (SERVPERF)

\section{حصوض الصواسم}

الحدود الموضوعية: ينم قياس جودة الخدمة الصحية من وجهة نظر المرضى بالمستثفى محل الدراسة. الحدود المكانية: أختيار مستشفى عين شمس الجامعى بالعباسية نموذج للمستثفيات الجامعية لأجراء البحث على المرضى المترددين عليها.

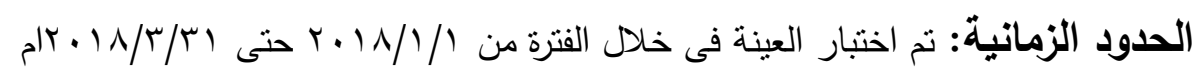

\section{السراسمة الميضانمية}

تهدف الدراسة لمعرفة تقييم المرضى للجودة الكلية للخدمة الصحية التي حصلوا عليها بالفعل ومعرفة مدى رضاهم عن الخدمة الصحية التى حصلوا عليها بالفعل من المستشفى

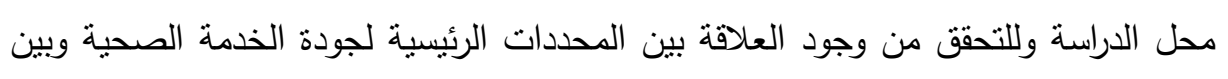

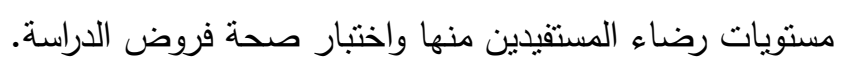
إجراءات الاراسة الميدانية: وحتى تحقق الباحثة هذه الأهداف، قامت بإجراءات تجميع وتصنيف بيانات الدراسة سواء النظرية أو الميدانية الأولية وتم فى هذه الدراسة استخدام الإحصاءت الوصفية لمتغيرات الدراسة:

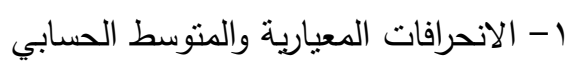
r- اعنماد ميزان تقديري وفقا لمقياس ليكارث الخماسي وليطي r- جدول ميزان تقديري وفقا لمقياس ليكارث الخماسي. 
اختبار فروض البحث وتحديد مدى قبول هذه الفروض من عدمة، فيما يتعلق بدور أبعاد

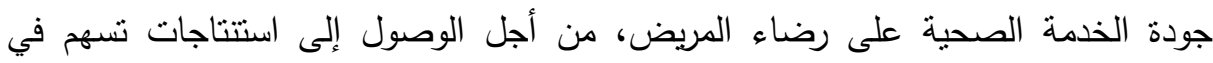

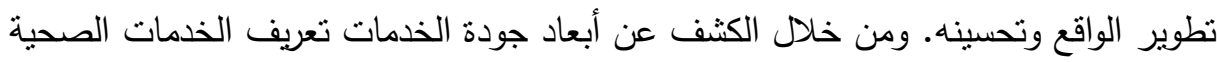
كما بدركها المرضى.

\section{هتغيوايت التراسمة}

تحتوي هذه الدراسة على نوعين من المتغيرات هما: 1- المتغيرات المستقلة: وهي المتغيرات التي تفسر الظاهرة محل الدراسة. وفي هذه الدراسة المتغيرات المستقلة تثتمل على: أبعاد جودة الخدمة، وتتكون من خمسة

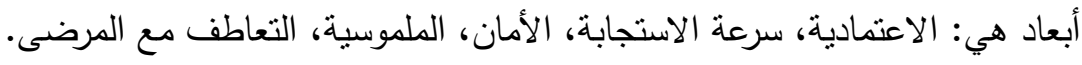
r-المتغير التابع: وهو عبارة عن المتغير الذي نرغب الباحثة عادة في التعرف على أسباب حدوثه والناتج المتوقع سواء من المتغير أو المتغيرات المستقلة.

وفي هذه الدراسة المتغير التابع هو: رضاء المريض عن جودة الخدمات المقدمة له.

\begin{tabular}{|c|c|c|c|c|c|}
\hline 0 & $\varepsilon$ & $r$ & Y & 1 & الرقم |متغيرات \\
\hline 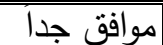 & 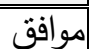 & إلى حد ما & غير موافق & غير موافق على الاطلات & \\
\hline
\end{tabular}
الجدول النالي يبين الإحصاءات الوصفية والانحرافات المعيارية لكل اسئلة الاستقصاء:

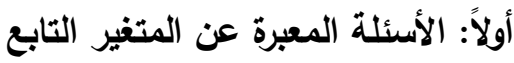

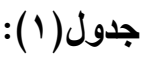

\begin{tabular}{|c|c|c|c|}
\hline الاتحراف المعياري & المتوسط الحسابي & المتغير التابع & المسلسل \\
\hline , 104 & 1,91 & التقدير العام للمستشفي & 1 \\
\hline
\end{tabular}

يتضح أن نتائج البحث الميداني لاتجاهات وآراء المرضى قد كثفت عن وجود قناعات منخفضه اتجاه التقدير العام للمستشفى (2,6:1,81) عدم الموافقة . 
ثانياً: الاسئلة المعبرة عن المتغيرات المستقلة

\begin{tabular}{|c|c|c|c|}
\hline الالمعرافي & المتوسط الحسابي & المتغيرات المستقلة & م \\
\hline, 12 & 2,26 عدم الموافقة & جودة الخدمة المقدمة من التمريض & 1 \\
\hline 15 & 2,39عدم الموافقة & جودة الخدمة المقدمة من الاطبّاء & 2 \\
\hline 12 & 1,82 عدم الموافقة & بيئة المستشفى اثثاء فترة اقامة المرضى & 3 \\
\hline 12 & 2,2 عدم الموافقة & خبرات المرضى اثثاء الاقامة في المستشفى & 4 \\
\hline, 15 & 2,63 موافق الى حد ما & تقييم المريض عند مغادرة المستشفى & 5 \\
\hline, 15 & 2,58 عدم الموافقة & معلومات عن المرضيى & 6 \\
\hline
\end{tabular}

يتضح أن نتائج البحث الميداني لاتجاهات وآراء المرضى قد كثفت عن وجود قناعات

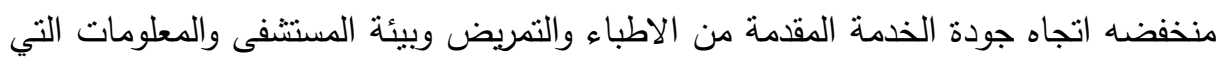
عبرت عن رائيهم في المستشفى كانت تتجه الى عدم الموافقة (2,6:1,81) . أما المتغير المستقل الخامس المعبر عنه تقييم المريض عند المند مغادرة المستشفى فكانت

$$
\text { موافقة الى حد ما (3,4:2,61). }
$$

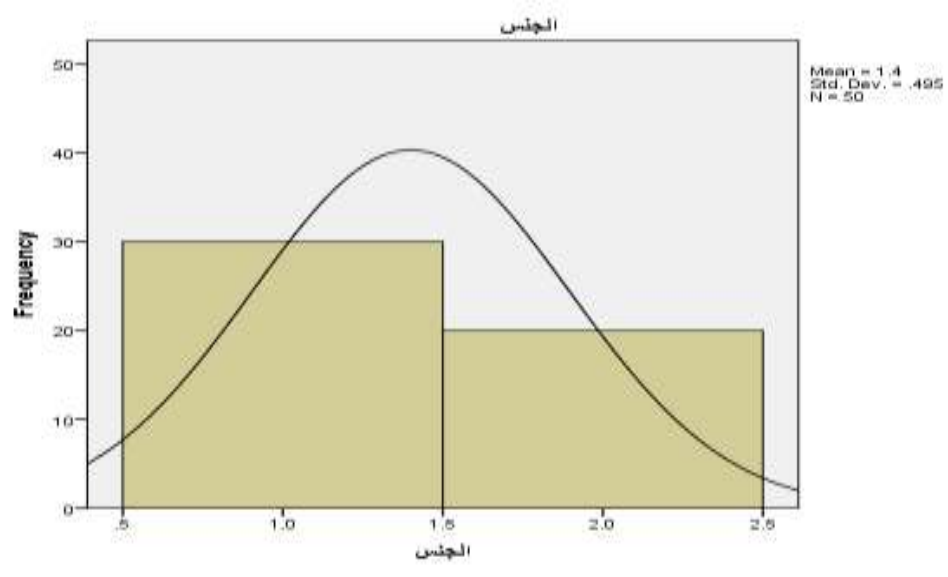

رسم توضيحي(1): يوضح المتوسط الجنس في العينة ان نسبة الرجال اكبر من النساء بما

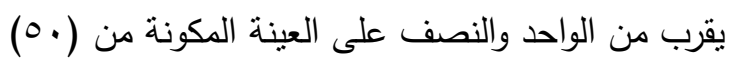




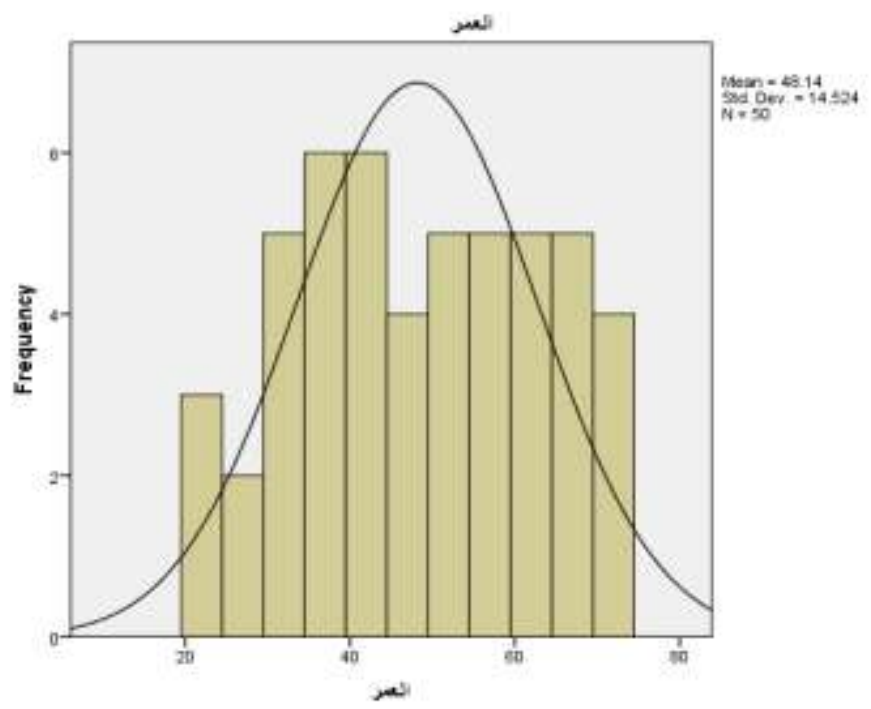

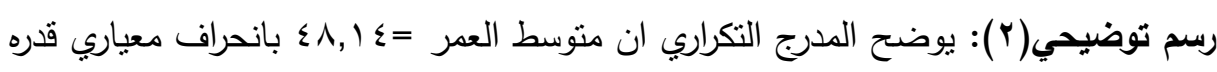

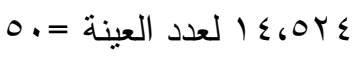

r-متغيرات الاستقصاء: احتوى الاستقصاء بعد استبعاد الثوابت كالاسم والتاريخ ومسلسل المريض على المتغيرات التالية

المتغيرات المستقلة وهى تعبر عن خمس فقرات تشمل على الى اسيؤال ومتغيرات تابعه وهى يعبر عنها سؤالين في فقرة واحدة وتتمنل في استجابة عينة الدراسة على فئى

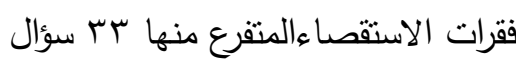
المعالجة الاحصائية المستخدمة: 1-الاحصاء الوصفي (المتوسط الحسابي والانحراف المعياري ). r-مقياس الصدق والثبات (Cronbach's Alpha).

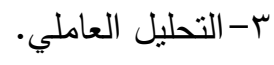
ع- الانحدار المتعدد. 
صدق وثبات الاداة: تم التأكد من الصدق الظاهري وصدق محتوى الاستقصاء باستخدام • الجامعي بجامعة عين شمس.

حساب ثبات الاداء بواسطة الحاسب الألى باستخدام معامل الفا كرو نباخ وكانت نتيجة

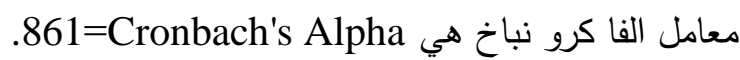
جدول(ץ): Reliability Statistics :

\begin{tabular}{|c|c|}
\hline Cronbach's Alpha & N of Items \\
\hline .886 & 28 \\
\hline
\end{tabular}

مما يعنى ثبات الأداة (الاستقصاء) وهذه نتيجة مقبولة لاعتبار أداة البحث ثابتة وقد تم

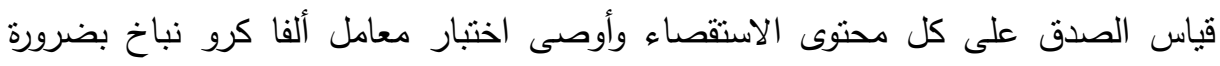

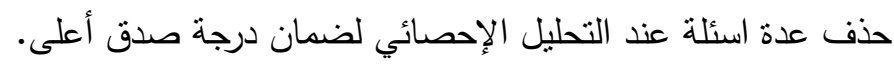

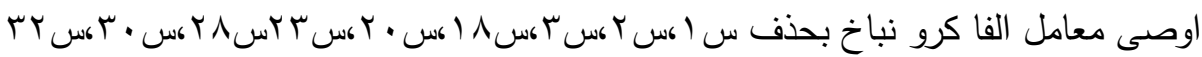
ويإجراء تحليل ثبات على باقي المتغيرات:

Reliability Statistics

\begin{tabular}{|c|c|c|}
\hline Cronbach's Alpha & $\begin{array}{c}\text { Cronbach's Alpha Base } \\
\text { on Standardized Items }\end{array}$ & N of Items \\
\hline \hline 905 & .908 & 23 \\
\hline
\end{tabular}

وعلية فان الاستقصاء اصبحت درجة عالية من الصدق اصبحت 905=Cronbach's. Alpha

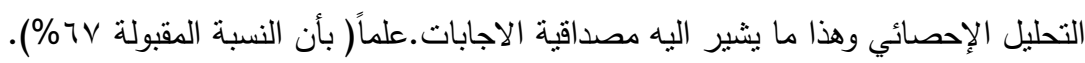

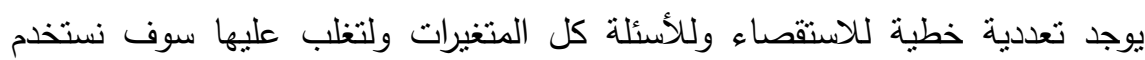

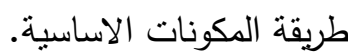

\begin{tabular}{|c|c|c|c|}
\hline \multicolumn{4}{|c|}{ Variables Entered/Removed a } \\
\hline Model & Variables Entered & Variables Removed & Method \\
\hline 1 & $\begin{array}{c}\text { REGR factor score } 2 \\
\text { for analysis } 2\end{array}$ & . & $\begin{array}{c}\text { Stepwise (Criteria: } \\
\text { Probability-of-F-to-enter } \\
<=.050, \text { Probability-of-F- } \\
\text { to-remove }>=.100) .\end{array}$ \\
\hline
\end{tabular}


Vوهو جدول يوضح اسماء المتغيرات التي ادخلت لمعادلة الانحدار وهو

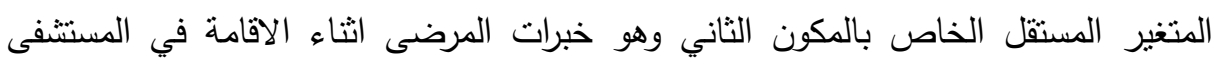

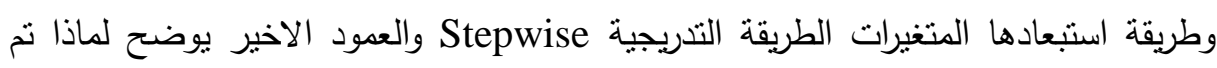
الاستبعاد سوف يتم استبعاد المتغيرات الاكبر من ا \$وسوف يتم ادخال المتغيرات التي قيمة Probability-of-F

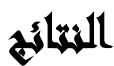

من خلال تحليل البيانات الميدانية يمكن عرض النتائج التالية:

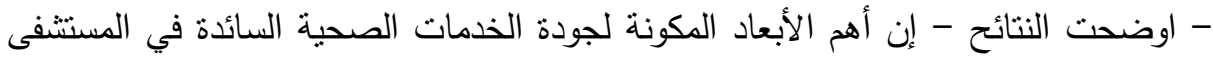
محل الدراسة والاكثر اهتماما وفقا لمقياس الأداء الفعلي (SERVPERF) هي على التوالي بعد التعاطف، بعد الأمان، بعد الاعتمادية، بعد سرعة الاستجابة، وبعد الجوانب الملموسة

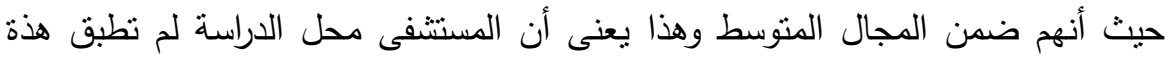
الابعاد كما ينبغى.

- وأن هناك اختلاف في تقييم المرضى لأبعاد جودة الخدمة الصحية ودرجة رضائهم وفقا للنوع والعمروالمستوى التعليمي، فمن المؤشرات المهمة والخاصة بالمريض أن العوامل مثل العمر والنوع والمستوى التعليمي تعتبر عوامل تؤثر على نسبة رضائه عن الخدمات المقدمة لهم. - ويوضح المنوسط الجنسى فى العينة ان نسبة الذكور اكبر من الاناث بما يقرب من الواحد

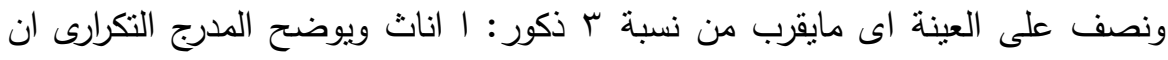

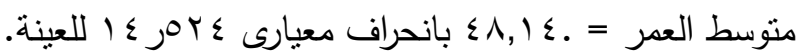
- يتضح من التوصل الى 0 عوامل يكون قيمة العينة ( الجذر الكامن) أكبرمن الواحد

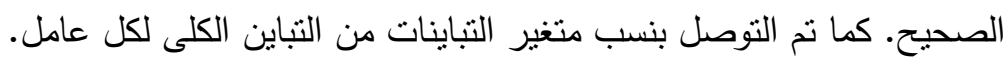
- عدد المجالات التى تم نتبعها أعلى من واحد صحيح بشكل تتازلى توزيع سب سؤال ل ل مجالات 


\section{نجد أن}

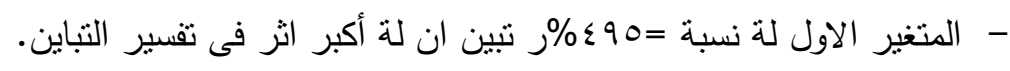

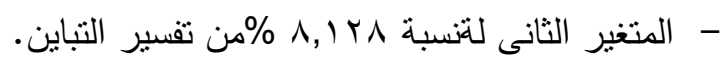

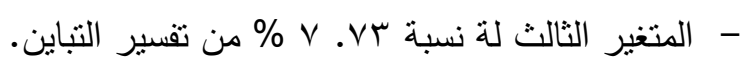

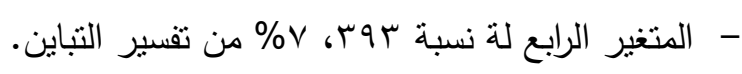

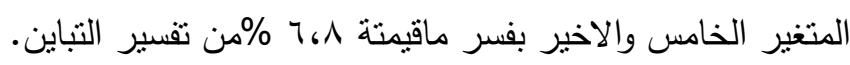

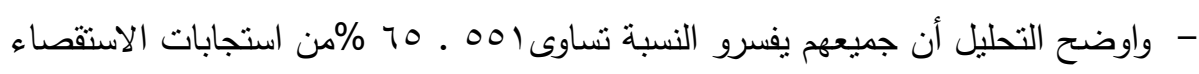

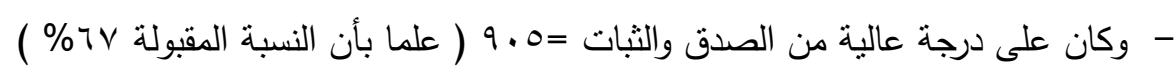
- توصلنا الى 0 عوامل نظرا لكون قيمتها العينية ( الجذر الكامن ) أكبر من الواحد الصحيح • كما تم التوصل الى نسب تفسير التباينات من التباين الكلى لكل عامل .

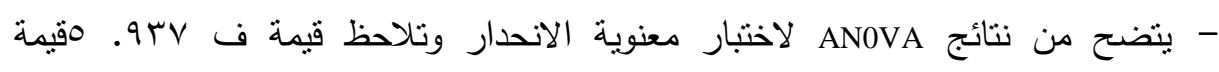

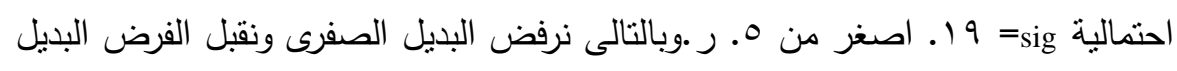

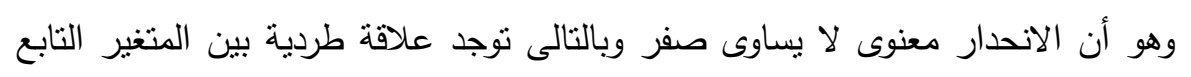

$$
\text { والمتغيرات المستقلة كلها . }
$$

- المتغير المستقل ذو دلالة إحصائية فى المعادلة وهو المكون الثانى فى معادلة الانحدار. - لا نوجد قيم منطرفة متعددة المتغيرات وهو شرط من شروط تطبيق تحليل الانحدار .

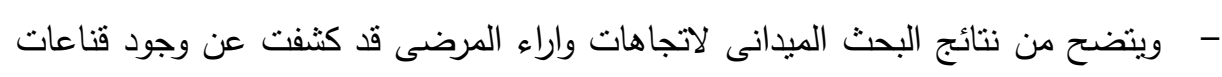
منخفضة اتجاة جودة الخدمة المقدة من الاطباء والتمريض وبيئة المستشفى ووجود علاقة التهاء

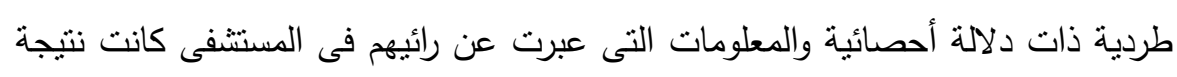

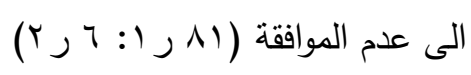

$$
\begin{aligned}
& \text { اختبار الفروض: }
\end{aligned}
$$

- يتضح من الاختبار لصحة الفرض الأول توجد علاقة ارتباط طردية ذات دلالة معنوية

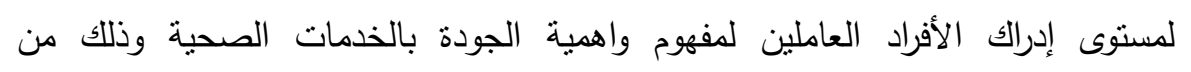

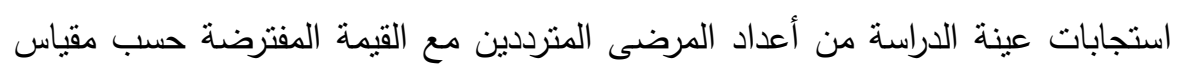


ليكرت الخماسي على مستوى جميع أبعاد جودة الخدمة الصحية، لذلك نرفض الفرضية الصفرية ونقبل البديلة حيث ان توجد علاقة طردية ايجابية.

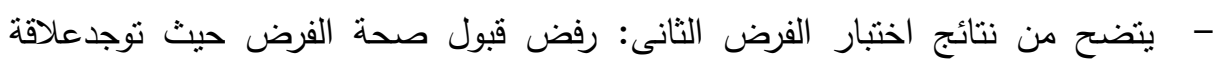

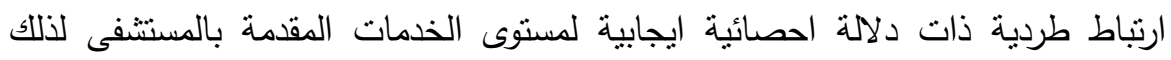
نرفض الفرضية الصفرية ونقبل البديلة حيث ان يوجد انخفاض بمسنوى الخدمات المقدمة دائه عن الخدمات التى تقدم بتطابق لمعايير الجودة .

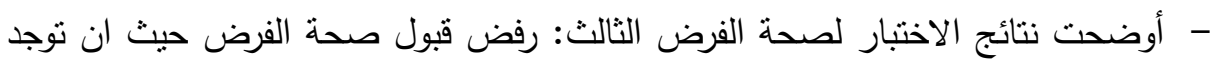

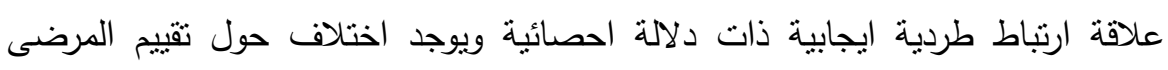

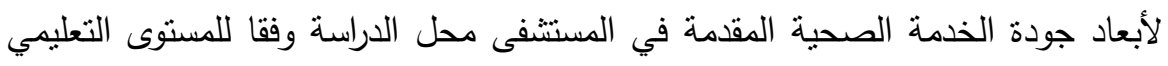
على جميع أبعاد جودة الخدمة الصحية مما يعني نرفض صحة الفرضية الصفرية وقبول

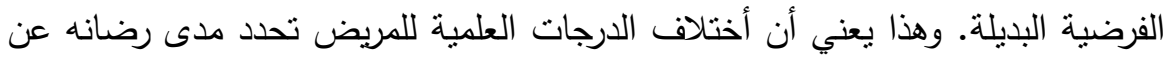

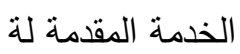
- - أن هناك علاقة ارتباط كبيرة بين أبعاد جودة الخدمة الصحية ورضاء المريض، ووجود علاقات معنوية عند مسنوى دلالة م.، والفرض، مما يعكس التأثير السلبى لأبعاد جودة إنباء الخدمة الصحية على رضاء المريض، وهو ما يتفق مع طبيعة الدراسة .

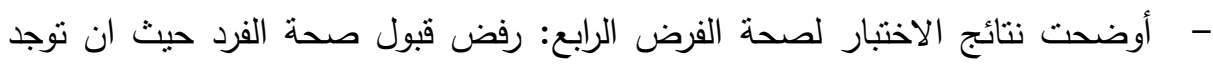

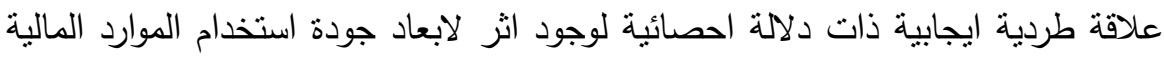
ووالاجهزة والمعدات ومستوى استخدام وتطبيق طرق وادوات الجودة مما يعنى رفض صحة

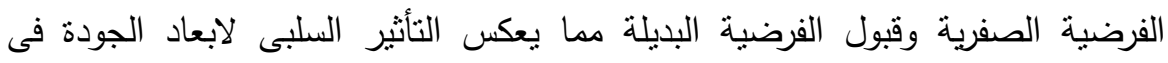
استخدام الموارد والاجهزة والمعدات. - أوضحت النتائج الاختبار لصحة الفرض الخامس توجد علاقة ارتباط طردية ايجابية ذات

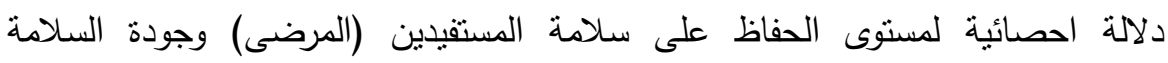
والصحة المهنية فى بيئة العمل بمقدار بr\% لابعاد الجودة لذلك نرفض الفرضة الصفرية 
ونقبل الفردالبديل حيث تعكس التاثير السلبى وهو انخفاض فى مستوى جودة الخدمة

\section{التوكياتي}

من خلال النتائج المستخدمة من هذة الدارسة التي نم التوصل إليها من الإطار النظري

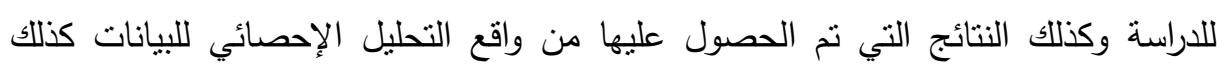
المقابلات الثخصبة نم التوصل الى التوصيات الاتية: - يتطلب فى الخدمات الصحية العمل بمعايير الجودة الثاملة وتطبيقاتها لرفع وزيادة الفاعلية ووالكفاءة تقليل الفاقد وتوفير التكاليف لمواجهة التحديات والوصول لتحقيق أهداف المستشفى ورضا المستقيدين (المرضى) بتقديم خدمات تفوق توقعاتهم وطموحاتهم، وتحقيق التنمية المستدامة. - العمل على زيادة المخصصات المالية وتوفير المستلزمات الطبية سواء كان ذلك من الأجهزة أم المعدات الحديثة من أجل تحسين جودة الخدمات الصحية المقدمة.

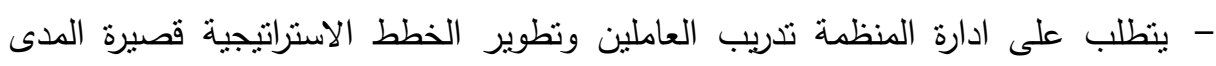
وطويلة المدى لتحقيق رغبات وطلبات المستفيدين واحتياجات السوق.

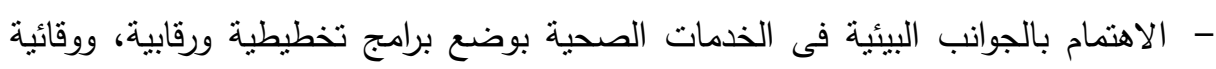
وحماية البيئة من التلوث والمخاطر الصحية وتحسين الصحة العامة للمجتمع.

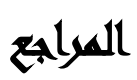

أحمد مجمد غنيم(9 . . r): إدارة الجودة الثاملة المكتبة المصرية للنشر والتوزيع، المنصورة

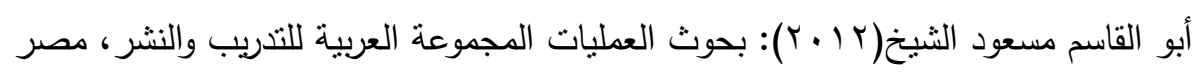

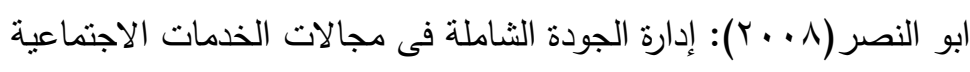
الحمدى طلال بن عابد(ع . . ؟): إدارة الرعاية الصحية، معهد الإدارة العامة المملكة العربية السعودية عابة

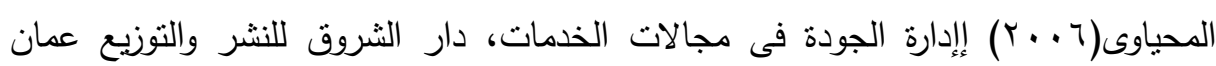

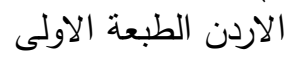




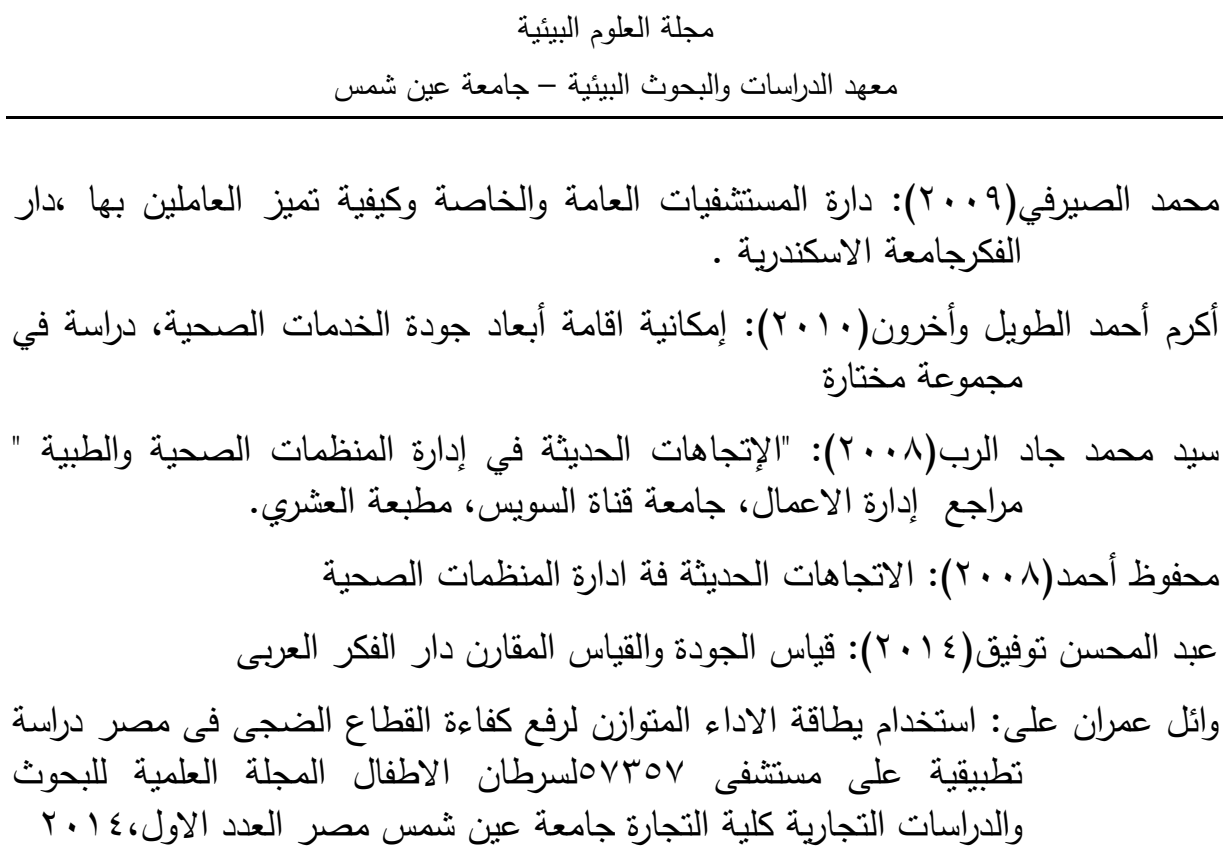

Onalenna, S. (2014): "Assessing Performance of Botswana'sPublic Hospital System: The Use of the World Health Organization Health System Performance Assessment Framework", Int J Health Policy Manag 3. Sep, pp. $179: 189 \mathrm{~s}$

Al-Qahtani, M., et al. (2012): Quality of care in accredited andnonaccredited hospitals: perceptions of nurses in the Eastern Province, Saudi Arabia. Journal of the Egyptian Public Health Association, 87 (3-4), 3944

Asadi, M., Tamburini, M. \& Gray, D. (2004): Patient'sneeds satisfaction, and health a

Jay H,Heizer,Barry Rerder,"operation management by Heizer prentice Hall,10th ed,ISBN,2003.

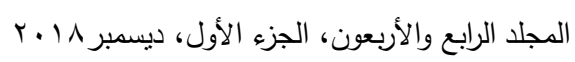




\title{
THE EFFECT OF QUALITY ON THE EFFICIENCY OF HEALTH SERVICES AN EMPIRICAL STUDY ON AIN SHAMS UNIVERSITY HOSPITAL
}

\author{
Barghout, Attiyat, N. ${ }^{(1)}$;Fanus, N. A. ${ }^{(2)}$ and Ragab, M. H. ${ }^{(3)}$ \\ 1) Ain Shams Hospital 2) Faculty of Commerce, Ain Shams university \\ 3) Institute of Environmental Studies and Research, Ain Shams \\ University
}

\begin{abstract}
The aim of this study is to know the extent to which the TQM approach is applied to the provision and promotion of the performance of health services, as well as to the extent to which the concepts of total quality can be used to improve the quality and efficiency of health services provided to clients in order to gain their trust. And the sample was randomized. The researchers used the descriptive analytical method by dividing the research into two parts. The first section: The theoretical approach illustrates the concept of quality, its principles, its distance and its resistance through Arabic and foreign books and periodicals

Section II: The practical approach includes a field study conducted at the Ain Shams University Hospital where the questionnaire was distributed as a tool for collecting data and information from the study community, where 100 copies were distributed to a random sample in the Department of Health Services and 50 copies were recovered after the complete answer. A variety of statistical methods have been used, including descriptive statistics, to describe the variables of the study, to show the effects of the study and statistical models to test the hypothesis of the study, and to indicate the accuracy of any of the models used
\end{abstract}




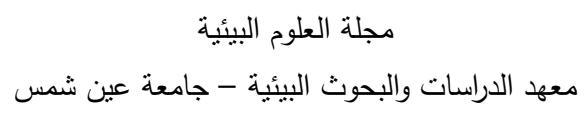

One of the main findings of the study is that there is a clear quality policy in the management of health services. The weakness of the organizational culture related to the concepts of Total Quality Management in the management of health services There is a lack of awareness of many of the administrative leaders of the Department of Health Services concept and the methodology of TQM. Lack of awareness of customers' needs and interests from all employees of the health services department. The absence of any effect on the quality of health services to the degree of satisfaction of the patient, which prevents them from being able to control the comprehensive quality program management of health services.

The study recommends that: To implement the overall quality standards and their applications by providing high quality services to meet the challenges and achieve the objectives of the hospital and the satisfaction of the beneficiaries to provide beyond their expectations and aspirations, and achieve sustainable development.

\section{Future studies:}

- Through the results of this study, it is possible to talk about some trends in studies that can be suggested in the future as follows:

- Obstacles and quality of service in public and private hospitals from the point of view of patients.

- Re-apply the field study to other service areas (schools, tourism, universities, banks, hotels).

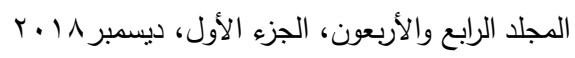

